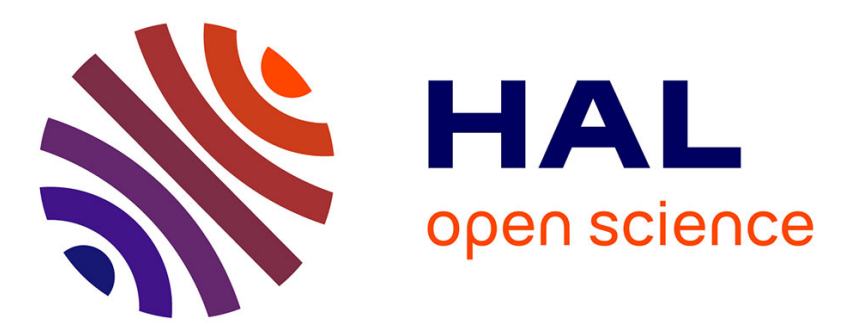

\title{
Les besoins éducatifs particuliers sont-ils un frein ou un levier dans la lutte contre les discriminations scolaires?
}

Hervé Benoit

\section{To cite this version:}

Hervé Benoit. Les besoins éducatifs particuliers sont-ils un frein ou un levier dans la lutte contre les discriminations scolaires?. Les Cahiers de la LCD, 2020, 3 (11), pp.61-83. 10.3917/clcd.011.0061 . hal-03149530

\section{HAL Id: hal-03149530 \\ https://hal-inshea.archives-ouvertes.fr/hal-03149530}

Submitted on 24 Feb 2021

HAL is a multi-disciplinary open access archive for the deposit and dissemination of scientific research documents, whether they are published or not. The documents may come from teaching and research institutions in France or abroad, or from public or private research centers.
L'archive ouverte pluridisciplinaire HAL, est destinée au dépôt et à la diffusion de documents scientifiques de niveau recherche, publiés ou non, émanant des établissements d'enseignement et de recherche français ou étrangers, des laboratoires publics ou privés. 


\title{
Les besoins éducatifs particuliers sont-ils un frein ou un levier dans la lutte contre les discriminations scolaires?
}

\author{
HERVE BENOIT
}

\begin{abstract}
Hervé Benoit est maître de conférences en sciences de l'éducation à l'INSHEA - université Paris Lumières et chercheur au laboratoire Grhapes (EA 7287). Il est notamment le coauteur de «La typologie des outils numériques dans le champ des EIAH à leur opérationnalité inclusive », La nouvelle revue de l'adaptation et de la scolarisation 78, 2545, 2017.
\end{abstract}

\section{Contact : herve.benoit@inshea.fr}

Résumé : Alors que les politiques publiques éducatives actuelles font de la prise en compte des besoins éducatifs particuliers des élèves en situation de handicap (ou non) le levier d'opérationnalisation de l'école inclusive, certains chercheurs considèrent que les besoins n'appartiennent pas au paradigme inclusif, en tant qu'ils renvoient à la compensation individuelle des déficiences et qu'ils participeraient paradoxalement d'un fonctionnement de discrimination scolaire en contribuant à la mise à l'écart des publics vulnérables dans des lieux éducatifs séparés. Dans un tel contexte institutionnel et scientifique, l'objet de cette contribution est de mettre en perspective les approches en présence pour tenter de répondre à la question de savoir en quoi les besoins éducatifs particuliers peuvent à la fois être considérés par les uns comme l'instrument d'un processus de discrimination, tandis que d'autres y voient au contraire le moyen le plus efficace de s'y opposer et de promouvoir l'école inclusive.

Mots-clés : Accessibilisation, Besoins éducatifs particuliers, Discrimination, École inclusive, Politiques publiques d'éducation.

\section{Introduction}

Les politiques publiques d'éducation en France ont fait depuis quelques années de « l'inclusion scolaire » $(2013)^{1}$, reformulée en «scolarisation inclusive» par la loi de $2019^{2}$, l'un des objectifs officiellement affichés et régulièrement relayés aussi bien dans les communiqués de presse du secrétariat d'État auprès du Premier ministre chargé des Personnes handicapées que dans les circulaires du ministère de l'Éducation nationale. Le 5 décembre 2017, est ainsi annoncé un plan comportant six grands axes pour engager une transformation «profonde et pérenne du système éducatif et médico-social » destiné à « permettre à l’École de la République

\footnotetext{
${ }^{1}$ L. $n^{\circ} 2013-595,8$ juill. 2013, d'orientation et de programmation pour la refondation de l'École de la République : $\mathrm{JO} \mathrm{n}^{\circ} 0157,9$ juill. 2013, p. 11379.

${ }^{2}$ L. n 2019-791, 26 juill. 2019, pour une École de la confiance : JO nº 0174, 28 juill. 2019.
} 
d'être pleinement inclusive » et à « offrir le droit à une scolarisation de qualité à tous les élèves en situation de handicap en répondant à leurs besoins éducatifs particuliers $»^{3}$. Le 17 juillet 2019, le ministre de l'Éducation nationale, Jean-Michel Blanquer, et la secrétaire d'État auprès du Premier ministre chargée des Personnes handicapées, Sophie Cluzel, installent le comité national de suivi de l'école inclusive, dans le cadre « des mesures constitutives du grand service public de l'école inclusive $»^{4}$, dont l'un des sept axes est « une école qui s'adapte aux besoins éducatifs particuliers des élèves $»^{5}$. Enfin, la circulaire de rentrée $2019^{6}$, en date du 5 juin 2019, est intégralement consacrée à la mise en place de l'École inclusive et annonce notamment que sera institué «dans chaque académie et dans chaque département un service public de l'École inclusive afin de ne laisser aucun élève au bord du chemin (...). Ce service a pour attributions (...) l'organisation, la mise en œuvre, le suivi et l'évaluation de la politique de scolarisation des élèves à besoins éducatifs particuliers, dont les élèves en situation de handicap ${ }^{7}$.

À travers l'affirmation de la loi du 8 juillet 2013 (art. 1) que le système éducatif «veille à l'inclusion scolaire de tous les enfants sans aucune distinction ", il apparaît que l'école inclusive n'a d'autre but que de prévenir toute discrimination relative au droit à une scolarité en milieu ordinaire. Il apparaît non moins clairement que le moyen principal de cette entreprise consiste, selon les sources citées, à répondre et à s'adapter aux besoins éducatifs particuliers des élèves concernés. En résumé, les politiques publiques éducatives actuelles s'assignent l'école inclusive pour but et présentent la prise en compte des besoins éducatifs particuliers des élèves en situation de handicap (ou non) comme le levier d'opérationnalisation de leur action.

Or la reconnaissance de la pertinence scientifique et de l'utilité sociale de la notion de besoins éducatifs particuliers est loin de faire consensus et certains chercheurs ont fait valoir qu'il s'agit là d'une prénotion, au sens de Durkheim, c'est-à-dire d'un concept issu de la pratique qui s'est formé « en dehors de la science et pour des besoins qui n'ont rien de scientifique » (Durkheim, 1977, p. 40). De surcroît, Ebersold et Detraux (2013), comme d'autres chercheurs, notamment en Italie (Vadalà et al., 2013 ; Ianes et Demo, 2013 ; Medeghini et al., 2012 ; D’Alessio, 2009), considèrent que les besoins n'appartiennent pas au paradigme inclusif, puisqu'ils se focalisent sur des déficiences à compenser et relèvent d'une approche diagnostique de l'élève pris isolément. En ce sens, l'évaluation des besoins participerait paradoxalement d'un fonctionnement de discrimination scolaire et contribuerait à la mise à l'écart des publics vulnérables dans des lieux éducatifs séparés (Ebersold et Dupont, 2019, p. 68).

Dans ce contexte institutionnel et scientifique troublé, l'objet de cette contribution est de mettre en perspective les approches en présence, à la lumière de la double analyse foucaldienne de la part généalogique et de la part critique du discours et de ses objets. Il s'agit, d'une part, de s'intéresser à la généalogie socio-historique du concept de besoins éducatifs particuliers (BEP) et, d'autre part, aux territoires d'énoncés dans lesquels il a pris et peut prendre des formes institutionnelles et épistémologiques variées. Plus précisément, la question à laquelle cette étude s'efforcera de répondre est celle de savoir en quoi les besoins éducatifs particuliers peuvent à la fois être considérés par certains comme l'instrument du processus de

\footnotetext{
3 http://handicap.gouv.fr/presse/communiques-de-presse/article/permettre-a-l-ecole-de-la-republique-d-etrepleinement-inclusive.

$4 \quad$ https://handicap.gouv.fr/presse/communiques-de-presse/article/jean-michel-blanquer-et-sophie-cluzelinstallent-le-comite-national-de-suivi-de.

${ }^{5}$ Ibid.

${ }^{6}$ Circ. $n^{\circ}$ 2019-088, 5 juin 2019 : BOEN n 23, 6 juin 2019, « Pour une École inclusive ».

${ }^{7}$ Ibid. (en gras dans le texte).
} 
discrimination des élèves en situation de handicap, tandis que d'autres y voient au contraire le moyen le plus efficace de s'y opposer et de promouvoir l'école inclusive.

\section{Cadre théorique de l'analyse des discours}

Les travaux de Michel Foucault permettent de s'affranchir du cadre de l'épistémologie du sujet pour prendre en compte la dimension du discours, en tant que producteur d'objets de savoir et vecteur de pouvoir. Parce qu'il a entrepris dans Les mots et les choses d'analyser les réseaux anonymes sous-jacents à l'histoire des discours, Michel Foucault a ouvert la voie de l'étude de la formation des discours, voie que nous empruntons ici dans le domaine de l'inclusion scolaire et particulièrement dans celui des besoins éducatifs particuliers.

De la leçon inaugurale au Collège de France prononcée le 2 décembre 1970, L'ordre du discours, publiée en 1971, se dégage un programme d'analyse du discours qui s'attache autant à la «part critique », qui cherche à établir les principes de fonctionnement d'un territoire d'énoncés en élucidant les relations qu'ils entretiennent entre eux, qu'à la «part généalogique », qui s'attache " aux séries de la formation effective du discours » et s'efforce d'identifier «son pouvoir d'affirmation»: «La part critique de l'analyse s'attache aux systèmes d'enveloppement du discours; elle essaie de cerner, de repérer ces principes d'ordonnancement, d'exclusion, de rareté du discours. [...] La part généalogique de l'analyse s'attache en revanche aux séries de la formation effective du discours : elle essaie de le saisir dans son pouvoir d'affirmation, [...] le pouvoir de constituer des domaines d'objets, à propos desquels on pourra affirmer ou nier des propositions vraies ou fausses. » (p. 71-72)

On s'inscrit ici dans une perspective de type poststructuraliste pour s'interroger sur la nature du ou des discours qui traversent les politiques publiques et sur lesquels celles-ci sont fondées. Ces soubassements discursifs, au sens que leur donne Michel Foucault, lorsqu'il évoque les épistémès, peuvent être définis comme le sol épistémologique envisagé sous l'angle des «configurations qui ont donné lieu aux formes diverses de la connaissance empirique » (Foucault, 1966, p. 13). Ces configurations restent impensées par ceux mêmes qu'elles déterminent; elles conditionnent le savoir et les pratiques institutionnelles et peuvent les assigner à des limites indépassables (Benoit, 2014c, p. 129). Elles orientent de manière déterminante « la forme et la signification $»^{8}$ (Benoit, 2003) des discours officiels et peuvent également intervenir dans la construction par les acteurs de leurs représentations à propos de l'inclusion scolaire et des élèves handicapés et des modalités de réponse à leurs besoins. Ces formations discursives peuvent s'articuler entre elles et se combiner avec d'autres éléments, dits et non-dits, pour constituer un dispositif stratégique, au sens foucaldien, c'est-à-dire une structure d'éléments hétérogènes caractérisés par la "prévalence d'un objectif stratégique », dont la fonction est de répondre à un moment donné à une urgence de type social ${ }^{9}$ (Foucault, 1977, p. 299). On sera ainsi en mesure de rendre compte, à travers l'étude généalogique de ces

\footnotetext{
${ }^{8}$ Forme et signification est le titre d'un essai de critique littéraire de Jean Rousset, paru en 1962 chez José Corti et constamment réédité depuis, dans lequel l'auteur forme le projet d'analyser « l'épanouissement simultané d'une structure et d'une pensée » (p. X). L'hypothèse est ici que la transposition de cette démarche dans le champ de la production d'écrits institutionnels est pertinente.

${ }^{9}$ Il peut s'agir, par exemple, de « la résorption d'une masse de population flottante qu'une société à économie de type essentiellement mercantiliste trouvait encombrante : il y a eu là un impératif stratégique, jouant comme matrice d'un dispositif, qui est devenu peu à peu le dispositif de contrôle-assujettissement de la folie, de la maladie mentale, de la névrose » (ibid.)
} 
discours (Foucault, 1966, 1971), des télescopages notionnels qui les caractérisent et d'ouvrir des pistes de compréhension de leur possible impact, d'une part, sur les ambiguïtés des politiques publiques et, d'autre part, sur les hybridations discursives qui les sous-tendent et peuvent en définitive influer sur les «déterminants généraux » (Sensevy, 2006, p. 219) des pratiques enseignantes.

\section{Apports d'une analyse socio-historique internationale à la généalogie du concept de BEP10}

L'un des facteurs déterminants de changement de pratiques au sein des systèmes éducatifs est assurément la formation initiale et continue dispensée à ses acteurs. Dans ce cadre, la volonté politique de rendre l'institution scolaire française plus inclusive se fait jour à travers la mise en place, à la rentrée 2017, d'une nouvelle formation des enseignants spécialisés conduisant à un diplôme intitulé certificat d'aptitude professionnelle aux pratiques de l'éducation inclusive (Cappei). Une fois encore, école inclusive et besoins éducatifs particuliers ont partie liée puisque le plus important (48 heures de formation) des cinq modules du tronc commun (144 heures) de cette nouvelle formation s'intitule «Identifier, analyser et prendre en compte les besoins éducatifs particuliers pour leur apporter des réponses pédagogiques et éducatives ». La question de la compréhension de cette notion mérite donc qu'on s'y attache, si l'on veut saisir en quoi elle peut être productive ou au contraire contre-productive au regard de la prévention et de la lutte contre les discriminations scolaires susceptibles d'être éprouvées par les jeunes en situation de handicap.

L'invention des special educational needs (dont la traduction officielle française est besoins éducatifs particuliers) par Mary Warnock, dans son rapport de 1978 rédigé à la demande du gouvernement britannique, et plus précisément du département de l'éducation et de la science (Department for éducation and science) ${ }^{11}$, répondait justement à la volonté de prévenir les discriminations scolaires conduisant à écarter de la voie ordinaire (mainstream) les élèves handicapés (handicapped children). L'idée avancée était même de renoncer au terme de handicap et de lui substituer celui de special needs, afin de s'éloigner de la logique médicale de la catégorisation des types de handicaps ou de déficiences (functional impairment), génératrice d'exclusion et d'éducation séparée. Les special educational needs étaient le moyen de s'en extraire en construisant un modèle plus pragmatique et moins stigmatisant. Comme le souligne Éric Plaisance (2012, p. 212), à l'occasion de la publication en 2010 de l'ouvrage collectif Special Educational Needs. A new look de Mary Warnock, Brahm Norwich et Lorella Terzi, l'intention explicite des rapporteurs de 1978, en forgeant cette notion, était de critiquer la pertinence de la distinction entre le normal et le handicapé (handicapped) et de promouvoir l'idée d'un continuum de special needs entre tous les types de difficultés d'apprentissage, sans chercher à rapporter certaines d'entre elles à une catégorie de déficience.

Dans cet esprit, la transversalité de la prise en compte des besoins dans le cadre d'une visée éducative universelle, englobant l'ensemble des élèves en difficulté, permet non seulement de concevoir l'inclusion en milieu ordinaire des enfants susceptibles d'être orientés dans une

\footnotetext{
${ }^{10}$ Cette partie reprend une partie des contenus développés dans un article publié en 2017 dans la revue Éducation comparée (REC).

${ }^{11}$ Ministère responsable de la politique d'éducation du Royaume-Uni. Aujourd'hui Department for education (DfE).
} 
filière spécialisée, mais aussi d'envisager la réorganisation du système d'enseignement dans une perspective de non-discrimination, par la mise en place des réponses adaptées en termes d'accompagnement et d'accessibilité. Cette approche, que l'on peut associer à l'idée que tout élève rencontre par définition des difficultés car elles sont l'enjeu même du processus d'apprentissage, s'oppose clairement à la logique des filières, fondée sur des procédures de catégorisation médico-psychologiques, consistant à diriger les élèves handicapés ou en difficulté dans des cursus séparés, considérés comme seuls capables de répondre de manière adaptée à leurs dysfonctionnements spécifiques (Benoit, 2008, p. 104).

Il apparaît dès lors que la prise en compte du continuum des besoins constitue un facteur déterminant pour l'émergence de pratiques scolaires de non-discrimination, en appui sur la notion d'accessibilité pédagogique et en relation avec une conception écologique, non médicalisée et scolairement située des difficultés d'apprentissage rencontrées par les élèves. Cette approche se présente comme le moyen de penser et de réaliser une école pour tous, sans recours à la catégorisation médicale et à l'enseignement séparé (Benoit, 2017, p. 23). Dans cette perspective nouvelle, le traitement pédagogique ou didactique, destiné à rendre accessible et par conséquent inclusive la situation d'apprentissage, se substitue au traitement institutionnel et curriculaire de la diversité des élèves.

Le concept de besoin va cependant entrer dans une longue période d'oubli institutionnel et épistémologique à la fin des années 1970 (ibid.). Il faudra en effet attendre 16 années pour que la Déclaration de Salamanque (UNESCO, 1994) les remette sur le devant de la scène éducative internationale. La préconisation clairement inclusive que comporte ce texte, qui affirme que « tous les élèves d'une communauté doivent apprendre ensemble, dans la mesure $\mathrm{du}$ possible, quels que soient leurs handicaps et leurs difficultés » (p. 11) s'appuyait logiquement sur l'opérationnalisation des special needs pour rendre le milieu scolaire apte à accueillir toute la diversité des élèves. Une véritable dynamique ascensionnelle entraîne alors ce concept résurgent dans les années qui suivent. Deux ans plus tard, en 1996, la création par la commission de 1'Agence européenne ${ }^{12}$ (European Agency for Development in Special Neeeds Education) l'installe durablement au cœur de la politique éducative de l'Union. Suivra dans le même mouvement le Forum mondial sur l'éducation de Dakar au Sénégal (UNESCO, 2000).

La raison de cet oubli est à chercher dans les conséquences de l'adoption par l'OMS, en 1980, de la classification internationale du handicap $(\mathrm{CIH})$, qui établissait au niveau mondial, pour deux décennies au moins, le modèle biomédical du couple du handicap-déficience comme référence institutionnelle, professionnelle et scientifique. Le processus de handicap, tel que décrit dans la $\mathrm{CIH}$, repose en effet sur une chaîne de causalité qui part de la déficience, définie comme perte de substance ou altération d'une fonction, de laquelle découlent des incapacités au plan fonctionnel, qui elles-mêmes génèrent des désavantages au plan social, c'est-à-dire des inconvénients en termes de vie sociale, d'insertion professionnelle, d'accès aux services de droit commun, etc. Une telle définition, fondée sur la référence à la causalité, sur la centration sur l'individu et sur la recherche de l'étiologie favorisait à l'évidence le recours au diagnostic et à la catégorisation médicale dans le champ éducatif. Elle ne pouvait qu'exercer une action délétère sur la notion de special educational needs, dans leur acception de continuum de diversité.

\footnotetext{
${ }^{12}$ Agence européenne pour le développement de l'éducation des personnes présentant des besoins éducatifs particuliers.
} 
Il en est résulté un infléchissement de la compréhension des special needs vers la référence au modèle médical conduisant à l'attribution du besoin à l'enfant, en tant que caractéristique personnelle. Dès 1978, le département de l'éducation et de la science avait d'ailleurs recommandé au comité présidé par Mary Warnock d'exclure du champ des special needs certains problèmes de santé comme la dyslexie. Une telle partition eut pour effet de casser le concept de besoins, de le fragmenter en divers types, relatifs à la cognition et aux apprentissages, au comportement, au développement émotionnel et social, à la communication, au développement sensoriel ou physique. Elle renvoyait ainsi à une origine supposée intrinsèque et constitutionnelle du besoin, pensé comme répercussion d'un dysfonctionnement interne inhérent à l'individu et elle accréditait l'idée que des mesures en termes de ressources spécialisées, de classes et d'écoles spéciales seraient nécessaires en fonction de la nature plus ou moins exigeante des besoins.

En France, dans le cadre de la loi du 30 juin 1975, l'usage qui est fait du terme besoin dans les textes réglementaires jusqu'à la fin des années 1990 semble ne correspondre à aucun modèle de pensée ou concept stabilisés et ne renvoie assurément pas à la conception développée par la commission Warnock. L'article 4 de la loi précise en effet que le choix de l'éducation spéciale s'opère «en fonction des besoins particuliers de chacun », ce qui fait des besoins un critère différenciateur, un outil de contrôle et de discrimination et non pas le moyen de concevoir un continuum entre tous les élèves.

Du côté de la Belgique (cmmunauté Wallonie Bruxelles) le décret du 3 mars 2004 a scindé l'enseignement spécialisé en huit types qui doivent être chacun, « adaptés aux besoins éducatifs généraux et particuliers des élèves relevant de l'enseignement spécialisé appartenant à un même groupe, besoins qui sont déterminés en fonction du handicap principal commun à ce groupe ». L'enseignement fondamental spécialisé est ainsi organisé en quatre « degrés de maturité » et non en cycles d'années d'études comme dans l'enseignement ordinaire (Delsarte, 2012, p. 37), aboutissant à la mise en place d'une organisation éducative dans un espace et une temporalité séparés, indexée sur un noyau défectologique postulé consubstantiel aux élèves concernés (Benoit, 2013, p. 55). Apparaissent dans cette législation des catégories de besoins qui se confondent avec les catégories de handicap et qui justifient ainsi l'existence de filières d'écoles spéciales séparées dédiées par différenciation structurale aux huit types d'enseignement spécialisé. Considérés comme répercussion de la déficience ou du trouble, les besoins se trouvent ainsi vidés de la valeur contextuelle et situationnelle, qui les émancipait de l'approche médicale, pour être repris et réintégré dans une logique de psychologisation et d'essentialisation du handicap.

En Italie, la loi-cadre $\mathrm{n}^{\circ} 104 / 1992$ de l'integrazione scolastica, encore en vigueur aujourd'hui, apparaît en rupture avec les politiques précédentes, qui s'enracinaient dans un cadre d'éducation spéciale et restaient centrées sur la déficience individuelle. Elle apportait un changement d'optique en se focalisant sur le milieu où se trouvent où les jeunes et pouvait faire ainsi transition avec les politiques ultérieures fondées sur les facteurs sociaux et environnementaux (Medeghini et D'Alessio, 2012, p. 16). Mais peu après la promulgation de la loi, est adopté le décret présidentiel de 1994 dit Atto di Indirizzo qui subordonne l'attribution d'un maître de soutien aux enfants handicapés à la délivrance d'un certificazione di handicap par une commission composée exclusivement de médecins ${ }^{13}$. Cette orientation ne sera pas modifiée par

\footnotetext{
${ }^{13}$ Dans le cadre de l'ASL (agence sanitaire locale), référence pour l'évaluation de l'état d'invalidité en vue de l'intégration des élèves à besoins éducatifs spéciaux à l'école (DPCM 185/2006).
} 
la nouvelle procédure (décret ministériel $n^{\circ}$ 185/2006), pourtant élaborée pour tenir compte du rôle de l'environnement, reconnu par la CIF (OMS, 2001), dans la production du handicap, mais qui « ne constitue pas une véritable tentative de remettre en question la catégorisation dans le cadre du processus d'intégration ${ }^{14}$ (D'Alessio, 2011, p. 11). Dans cette perspective, les besoins éducatifs particuliers restent liés à la catégorisation médicale des troubles et sont en conséquence utilisés, au même titre qu'un taux d'incapacité, comme critère d'attribution de ressources à des élèves handicapés. Le paradoxe de la situation italienne à cet égard est qu'une compréhension individuelle et médicale des besoins coexiste avec une organisation inclusive du système éducatif, puisque tous les enfants sont accueillis de droit dans le milieu ordinaire depuis la loi n 577 de 1977. Au-delà de la scolarisation séparée, des discriminations de l'intérieur peuvent néanmoins être repérées et l'on peut tenter l'hypothèse, en appui sur le concept de «dispositif stratégique » développé par Foucault (1977, p. 299) que le transfert d'une masse de population de jeunes, naguère accueillie et éduquée dans des établissements de santé, vers des établissements scolaires, dans une logique d'unification de lieux éducatifs auparavant séparés dans l'espace, s'accompagne de la réinstallation sous le toit commun de cloisonnements symboliques, aux plans du langage et des représentations, aux fins de produire des modes d'affiliation sociale distincts (Benoit, 2014a, p. 190). Cette hypothèse pourrait également permettre de rendre compte de la distorsion de certaines pratiques affichées comme inclusives, dans le système éducatif français, bien que consistant à faire fonctionner des dispositifs d'appui à la non-discrimination scolaire (Ulis) ${ }^{15}$ comme des classes spéciales ségrégatives.

\section{Apports d'une analyse critique des territoires discursifs dans le champ des BEP'16}

\subsection{Les discours de pathologisation du social : une forme de cécité sociale}

Historiquement, l'une des premières séries de discours est celle de la pathologisation des difficultés sociales. Elle a été très largement documentée, à partir de 1990 par les travaux de Jacqueline Gateaux. Cette auteure a montré comment, au début des années 1960, le discours savant sur la « débilité légère » s'est développé comme une construction idéologique répondant à un problème social : «le masque psychologique d'une réalité sociologique : la résistance ou la non-adhésion aux normes culturelles de l'école » (Gateaux, 1990).

L'approche psychopathologique de l'échec scolaire est utilisée alors comme caution médicale aux pratiques de marginalisation par une école frappée de cécité sociale à l'égard des inégalités (Bourdieu, 1979). Dans ce domaine, c'est une logique de type nosographique qui prévaut et qui se met au service d'un système qui produit nécessairement des laissés-pour-compte, vis-à-vis desquels l'échelle sociométrique (Gateaux, 2003) se révèle efficace, «parce que ce dispositif répond aux mécanismes de distanciation sociale consécutifs à la démocratisation de l'enseignement et contribue à résoudre les problèmes d'une société productiviste en pleine expansion » (Gateaux, 2005, p. 43).

\footnotetext{
14 «Does not constitute a real attempt to question of categorisation at the standpoint of the process of integrazione ».

${ }^{15}$ Unités localisées pour l'inclusion scolaire (MEN 2010, 2015)

${ }^{16}$ Cette partie reprend une partie des contenus développés dans un article publié en 2014 dans La nouvelle revue de l'adaptation et de la scolarisation (NRAS, $\left.\mathrm{n}^{\circ} 65\right)$.
} 
Jusqu'au début des années 1990, se sont ainsi trouvés réunis, dans un continuum de débiles, les élèves issus de l'immigration et appartenant aux milieux les plus faibles économiquement (personnels de service, manœuvres), tous naufragés de l'échec scolaire (notion qui se développe au début des années 1970).

En 1980, l'Organisation mondiale de la santé (OMS) procède à un remaniement significatif des frontières traditionnelles de la déficience intellectuelle. Dans le cadre des «déficiences de l'intelligence », la dénomination du «retard mental » remplace celle de la «déficience intellectuelle »; l'échelle des «insuffisances » en référence au QI subsiste, mais les limites correspondant aux différents degrés de ce retard (profond, sévère, modéré ou léger) sont rééchelonnées à partir d'une nouvelle approche de ce que l'on nommait "débilité légère ». Cette évolution n'est prise en compte qu'au début de l'année $19899^{17}$. À compter de cette date, l'ensemble des élèves dont le QI est compris entre 70 et 80/90, qui étaient auparavant étiquetés débiles légers et orientés vers des filières spéciales, ne sont plus considérés comme déficients. La composante de construction historique et sociale de la notion de normalité apparait ici de manière évidente (Benoit, 2005, p. 95).

On aurait pu penser qu'une telle réforme, en disqualifiant le discours psychologique justificatif, allait briser le cycle de la filiarisation dans des structures ségrégatives d'élèves en difficulté scolaire issus de milieux populaires. En fait, dans nombre de départements, on se contente dans les années 1990 de changer la dénomination de la classe de perfectionnement en classe d'intégration scolaire (Clis) sans en modifier le recrutement. Dans le second degré la poursuite de la ségrégation des jeunes issus des milieux les plus défavorisés dans les Segpa des collèges s'appuie sur un discours substitutif, celui de la « grande difficulté scolaire ${ }^{18}$ ou «difficulté scolaire grave et durable » : à la pathologisation du problème succède la gradation scolaire de l'écart, qui constitue une forme connexe de catégorisation normative non plus clinicocorrective, mais scolaro-corrective. Aujourd'hui encore, les inégalités sociales apparaissent de manière criante lorsqu'on compare les populations d'élèves de Segpa à celles des classes ordinaires de collège.

\subsection{Les discours médicaux de remédiation du trouble : une forme de cécité pédagogique}

Dans la continuité du discours de pathologisation du social, s'est développé un discours médical de remédiation du trouble, qui postule que les difficultés d'apprentissage sont d'origine neurobiologique et découlent, dans une logique substantialiste, d'un trouble constitutionnel ou d'un dysfonctionnement primaire (par exemple les troubles « dys »).

Ce type d'approche de type tautologique consistant à dire d'un élève qui manifeste de la lassitude dans ses apprentissages qu'il serait fatigable, voire atteint d'un syndrome de fatigue chronique $^{19}$ (SFC) ou d'un déficit attentionnel moteur et/ou perceptif ${ }^{20}$ (DAMP), détourne l'attention de ce que Gérard Vergnaud appelle « l'analyse de l'activité, dans ses relations avec

\footnotetext{
${ }^{17}$ A., 9 janv. 1989, portant « Nouvelle nomenclature des déficiences, incapacités et désavantages ».

${ }^{18}$ Formule de la circulaire princeps du 6 février 1989, qui transformait les SES en sections d'enseignement général et professionnel adapté (Segpa), et reprise sous des formes proches en 1996 et 2006 (Circ. n 2006-139, 29 août 2006).

${ }^{19}$ Ce syndrome a été identifié dans les pays anglo-saxons au milieu des années 1980. L'Organisation mondiale de la santé le considère comme une maladie neurologique.

${ }^{20} \mathrm{Ou}$ ADD/ADHD (Attention deficit hyperactivity disorder). Diagnostic souvent posé dans le contexte suédois (Claes Nilholm et Christer Hakanson, 2003).
} 
la structure des situations offertes et avec les conditions affectives, sociales et institutionnelles dans lesquelles prend place cette activité » (Vergnaud, 2004, p. 8).

C'est cette même approche que l'on retrouve communément dans le champ de la dyslexie et plus généralement dans celui des troubles spécifiques du langage oral et écrit (TSL). La circulaire du 31 janvier $2002^{21}$ commence en effet par reconnaitre l'existence d'une déficience liée à un dysfonctionnement primaire du système neurologique central ${ }^{22}$ (c'est-à-dire d'origine congénitale, et donc sans aucun lien ni avec l'environnement social et scolaire, ni avec une déficience mentale, sensorielle ou motrice, ni avec des perturbations acquises avant ou peu après la naissance ou des traumatismes postérieurs) avant d'aborder la question des parcours scolaires et des modalités de prise en charge. On se situe clairement dans une perspective où la réponse aux besoins éducatifs particuliers est subordonnée à la pose d'un diagnostic : « La qualité du repérage, du dépistage et du diagnostic (...) constitue une étape essentielle et déterminante pour définir les meilleures conditions d'une prise en charge individualisée » (Circ. $n^{\circ}$ 2002-024, 31 janv. 2002, Mise en œuvre d'un plan d'action pour les enfants atteints d'un trouble du langage oral ou écrit).

Dans son ouvrage Ni fou, ni gogol! Orientation et vie en ITEP, Hugo Dupont (2016) décrit des mécanismes sociaux et langagiers du même ordre dans le cadre de l'orientation en institut thérapeutique, éducatif et pédagogique (ITEP) d'élèves considérés comme atteints de troubles psychiques affectant fortement leur comportement scolaire.

\subsection{L'effet d'un discours catalyseur : rencontre des discours inclusifs et des discours médicaux}

Que l'on se réfère à la définition de Rutherford (2012) ou de Slee (2006), l'inclusion se réalise lorsque «tout le monde a sa place », lorsque «tout le monde est là »: Who's in ? Who's out ? and How come ${ }^{23}$ ?

S'appuyant sur les travaux d'Armstrong et de Barton, D'Alessio (2008, p. 36), considère l'inclusion « comme un processus de transformation des systèmes éducatifs et des cultures qui permet à tous les élèves de participer entièrement et équitablement au processus d'apprentissage dans les classes ordinaires ». Elle indique également que «le développement de l'éducation inclusive entraîne une déconstruction des notions actuelles de scolarisation, d'apprentissage et d'éducation afin d'établir les préconditions de l'apprentissage de tous » (ibid., p. 44).

Le discours catalyseur de la rencontre entre les discours de type médical et les discours inclusifs est le discours institutionnel injonctif de collaboration qui, en France et en Europe, s'est progressivement imposé à tous les acteurs et selon lequel la réussite du processus d'inclusion est subordonnée au développement d'une collaboration efficace entre l'école et les ressources externes de toutes sortes - principalement médico-sociales, médicales et familiales - gravitant autour d'elle. En France, par exemple, le décret $n^{\circ} 2009-378$ du 2 avril 2009 érige la «coopération entre le secteur médico-social et les établissements scolaires » en exigence institutionnelle.

\footnotetext{
${ }^{21}$ Circ. $n^{\circ}$ 2002-024, 31 janv. 2002, Mise en œuvre d'un plan d'action pour les enfants atteints d'un trouble du langage oral ou écrit.

${ }^{22}$ Le National Joint Comitee On Learning Disabilities définit la dyslexie en 1987 comme « un trouble intrinsèque à l'individu et présumé secondaire à un dysfonctionnement du système nerveux central », (« Learning disabilities : issues of definition », Journal of Learning Disabilities, $\mathrm{n}^{\circ}$ 20, pp. 107-108).

${ }^{23}$ Que l'on peut traduire par « Qui est là ? Qui n'est pas là ? Et pourquoi ? »
} 
L'installation, dans le champ des pratiques pédagogiques et éducatives, mais aussi dans celui des sciences de l'éducation, du concept de trouble et des catégorisations nosographiques correspondantes, portés par le discours médical dominant de répercussion/remédiation de la $\mathrm{CIH}^{24}$ (OMS, 1980) a conduit à croiser la notion du trouble et celle de besoins éducatifs particuliers. Du choc de ces deux objets noyaux de discours, il est résulté une fission et une hybridation du concept des besoins, qui dès lors se trouvent le plus souvent conçus comme intrinsèques à l'élève concerné, puisqu'ils équivaudraient à la compensation de la répercussion de son trouble, c'est-à-dire à son envers positif. La logique du trouble renforce ainsi l'idée que la connaissance du diagnostic et des invariants qui le caractérisent aurait a priori vocation à déterminer à la fois la nature des besoins et le choix des mesures adaptées (Benoit, 2014b, p. 197). Cette même logique contribue de surcroît à occulter les déterminants sociaux qui sont en quelque sorte fondus dans un discours des besoins conçus comme intrinsèques à la personne, consécutifs à sa pathologie et par là même essentialisés. Ainsi cécité pédagogique et cécité sociale se trouvent-elles combinées.

\section{Discussion et conclusion : intérêt d'une approche transdisciplinaire}

Selon Éric Plaisance (2013, p. 219), la notion de besoins éducatifs particuliers est controversée, car considérée par plusieurs auteurs comme « egocentrée » (Ebersold et Detraux, 2013, cité par Plaisance) et plus proche de la catégorisation médicale des troubles et des déficiences que de l'accessibilité pédagogique, qui, quant à elle, met l'accent sur l'impact de l'environnement scolaire plutôt que sur les caractéristiques déficitaires intrinsèques des élèves. On peut considérer dans cette perspective que les besoins éducatifs, parce qu'ils présupposent un modèle de compréhension interne à l'apprenant et qu'ils « sont toujours attribués à l'enfant », en rapport avec son dysfonctionnement individuel et sans tenir compte du rôle du contexte dans leur émergence (D'Alessio, 2009, p. 44), n'appartiennent pas au paradigme inclusif, en tant qu'ils renvoient à la compensation individuelle des incapacités, conduisent à la neutralisation des contextes et relèvent d'une approche diagnostique de l'élève pris isolément (Vadalà, Medeghini et D'Alessio, 2013 ; Ianes et Demo, 2013 ; Medeghini et D’Alessio, 2012). Ainsi la focalisation sur les besoins éloignerait-elle les acteurs de l'éducation d'une approche «polycentrée », où le contexte éducatif est analysé comme un intersystème et où le regard se porte «sur le devenir de la personne et l'effet capacitant des pratiques » (Ebersold et Detraux, 2013, p. 113). Érigée en tant qu' " acte solennel de catégorisation, qui tend à produire ce qu'il désigne », l'évaluation des besoins, instituée par les politiques publiques en vue de la concrétisation du droit à la scolarisation des jeunes handicapés, via l'outil réglementaire du Géva-sco, se présenterait alors paradoxalement comme un processus participant des mécanismes sociaux par lesquels s'opère l'institution des différences sociales entre des populations d'élèves en leur attribuant des identités relatives à leur scolarisabilité ou au contraire à leur assignation à des espaces éducatifs et curriculaires séparés (Ebersold et Dupont, 2019, p. 67). Dans ces conditions, les besoins fonctionnent comme un critère différenciateur construit à partir de la mesure « d'une distance scolaire objectivée par l'intensité du soutien requis » (ibid., p. 76), c'est-à-dire d'un écart à la norme socioculturelle, autrement dit à la forme scolaire, entre les jeunes considérés comme capables ou, au contraire, incapables de se comporter d'une manière conforme à l'ordre de l'École.

\footnotetext{
${ }^{24}$ Classification internationale du handicap, en vigueur jusqu'en 2001.
} 
L'analyse des soubassements épistémiques, des recouvrements de territoires discursifs et des collisions d'objets conceptuels présentée plus haut permet de rendre compte de cette hybridation du concept de special needs (Benoit, 2017, p. 34). On peut faire 1'hypothèse que cette double valence notionnelle a été initialement, et continue d'être, soutenue par la transposition dans le lexique de l'éducation inclusive d'éléments de discours issus du domaine médico-psychologique et qui se transforment en éléments de langage (au sens politique) dans la mesure où ils sont le moyen d'un positionnement statutaire du professionnel et le signe d'un pouvoir, d'un «titre de noblesse culturelle» (Bourdieu, 1979), qui lui sont conférés. En évoluant, dans plusieurs pays européens, vers des centres de ressources pour l'école ordinaire les institutions spécialisées (Agence européenne pour le développement de l'éducation des personnes ayant des besoins particuliers, 2003, pp. 15, 19, 28) ont en effet importé dans le système scolaire, dans ses pratiques et dans les études dont elles font l'objet un vocabulaire relatif aux catégories diagnostiques des pathologies (autisme, psychose, états limites...) et aux symptômes comportementaux dépression, frustration, hyperactivité, violence..., ou encore faisant référence au mal-être et à la souffrance (Emery, 2014). L'utilisation dominante d'un tel discours médico-psychologique a non seulement pour effet de laisser à l'écart la question des savoirs scolaires, mais encore d'induire une médicalisation du processus inclusif au détriment des approches pédagogiques et éducatives. Un concept, comme celui de l'évaluation connaît dans ce contexte une très forte ambiguïsation qui tend à obérer, quand il s'agit de l'éducation d'élèves handicapés le versant des acquis et des progrès scolaires pour privilégier celui des besoins de type psychothérapeutique et des services de soins susceptibles de les satisfaire.

En 2007, l'Agence européenne avait précisément engagé une recherche internationale sur la question de l'évaluation des besoins en contexte d'éducation inclusive (Watkins, 2007). Les attendus de ce projet étaient de déterminer, en s'appuyant sur des experts issus des 23 pays représentés, les conditions dans lesquelles les systèmes éducatifs pouvaient s'orienter « vers un mode d'évaluation inclusive » (p. 49).

L'une des principales conclusions de ces travaux fut de mettre en lumière que les pratiques évaluatives concernant les jeunes handicapés consistaient le plus souvent à identifier, dans une optique réparatrice ou compensatrice, les besoins en matière d'interventions rééducatives ou thérapeutiques destinées à remédier à leurs dysfonctionnements et à les réduire, au détriment d'une évaluation dans le cadre scolaire des besoins du point de vue des apprentissages. Le risque de ségrégation induit par l'inspiration médicale d'une telle approche centrée sur le déficit était souligné et la démarche préconisée était de prendre en considération le contexte dans lequel apparaissent les difficultés de l'élève. Dans cet esprit, les actions de soutien à l'apprentissage, en appui sur l'évaluation des forces et ressources de l'apprenant, étaient considérées comme préférables au recours à des mesures spéciales, voire à l'orientation vers des filières éducatives séparées.

C'est cette logique des besoins-trouble de santé qui est documentée, d'un point de vue sociologique (Ebersold et Dupont, 2019), en tant qu'elle a été instrumentalisée de longue date par les politiques publiques comme outil de gestion des flux d'élèves et critère différenciateur entre éducation ordinaire et spécialisée, et parallèlement configurée, dans le même but, en besoins-trouble à l'ordre scolaire.

On a vu néanmoins se développer au cours des 10 dernières années, notamment dans le cadre du réseau de chercheurs de l'OPHRIS et de la prise en compte de l'accessibilité didactique, une nouvelle approche des «besoins situés» (Benoit, 2014b, p. 197), qui s'émancipe 
progressivement de la logique médicale et qui tend à se décentrer de la réponse compensatoire aux répercussions supposées du trouble. L'idée est que « des besoins situés peuvent ainsi être identifiés par les enseignants qui peuvent ensuite adapter les situations à ces besoins. Certes des analyses a priori des besoins des élèves handicapés et des situations d'enseignement sont nécessaires, mais il s'agit plutôt de pouvoir mettre en place un processus dialectique entre analyse de besoins et analyse de situations » (Assude, 2019, p. 17). Cette approche s'appuie à la fois sur le fait que le pathologique et le normal ont beaucoup de traits en commun et que les compétences qui font défaut à un sujet sont toujours fortement liées à des registres particuliers de l'activité et de l'expérience (Vergnaud, 2004, p. 9) et beaucoup moins à ce que l'on peut savoir de la nature du trouble ou des caractéristiques individuelles de la personne. Un élève rencontre un obstacle dans ses apprentissages ou dans sa vie familiale ou sociale, il en résulte un besoin de médiation qui est précisément constitutif du besoin éducatif particulier (Benoit, 2008, p. 102). Ce besoin éducatif, que l'on pourrait désigner par le terme de besoin-obstacle n'est donc pas considéré comme constitué préalablement à la situation d'enseignementapprentissage, il est le produit des interactions qui la caractérisent ; il n'est pas intrinsèque à l'élève. Il s'agit dès lors pour l'enseignant d'ajuster l'accessibilité des tâches d'apprentissage à partir de l'observation de leur déroulement effectif en situation. En s'organisant autour des obstacles auxquels l'élève se heurte dans son environnement scolaire et sur lesquels l'enseignant intervient dans une visée d'accessibilisation de cet environnement, une telle démarche a vocation à s'instituer comme un élément fort de pratiques professionnelles enseignantes de non-discrimination. Elle s'inscrit de fait dans la conception universelle (ONU, 2006) dans la mesure où les besoins repérés en situation, ou besoins situés, pourraient fonctionner comme un incubateur d'accessibilisation du contexte d'apprentissage pour tous, prévenant ainsi tout risque de stigmatisation et de ségrégation.

C'est cette même approche que développe Thomazet (2012, p. 16), lorsqu'il subordonne « le passage à l'école pour tous, dite inclusive » à la capacité du système éducatif à « remettre sur le terrain de l'école le concept de besoins éducatifs particuliers ». Se référant aux recommandations du rapport Warnock, il préconise de prendre en compte «les besoins des élèves à l'école et non plus l'origine de leurs difficultés » (ibid., p. 11). Il ajoute que le concept de besoins pourrait constituer un puissant levier de changement s'il s'appliquait non plus aux élèves, mais à l'école elle-même : «Passer des besoins de l'enfant aux besoins de l'école nécessite de dépasser le paradigme intégratif (...) pour s'interroger sur l'organisation de l'école afin de la rendre à même de répondre aux besoins de tous les élèves qui la fréquentent » (ibid. p. 16).

En définitive, les approches généalogique et critique du discours des besoins, successivement développées pour répondre à la question initiale, apparaissent convergentes et tendent à montrer que la double face du concept de BEP permet de rendre compte d'un paradoxe apparent, qui ne renvoie pas à un antagonisme ou à une contradiction épistémologiques, mais plutôt au choix d'un angle de vue en partie déterminé par le cadre scientifique d'analyse : le cadre sociologique s'attache à mettre à jour la dimension différenciatrice et discriminante des BEP, en tant qu'ils participent objectivement des mécanismes sociaux d'exclusion, celui des sciences de l'éducation s'intéresse au contraire aux qualités opérationnelles de l'autre face de ce même concept, au sens où il permet aux enseignants de penser et de réaliser une école accessible tous, sans recours à la catégorisation médicale et à la ségrégation scolaire, en référence avec une conception écologique et scolairement située des difficultés d'apprentissage rencontrées par les 
élèves. Lever cette ambiguïté pourrait aussi aider les praticiens à surmonter les dilemmes auxquels ils sont confrontés dans l'exercice de leur mission inclusive.

\section{Bibliographie}

Assude T. (2019), «Dynamique inclusive, don et reconnaissance », in (Perez J.-M., Benoit H. et Suau G. (dir)), "Recherche en éducation et pratiques inclusives, La nouvelle revue Éducation et société inclusives, $\mathrm{n}^{\circ} 86, \mathrm{pp}$. 13-26.

Agence européenne pour le développement de l'éducation des personnes ayant des besoins particuliers (2003), Les besoins éducatifs particuliers en Europe, disponible en ligne: www.european-agency.org/sites/default/files/special-needs-education-ineurope_sne_europe_fr.pdf

Benoit H. (2017), «Integrazione scolastica en Italie special needs en Angleterre et inclusion scolaire en France : convergences et divergences dans le contexte européen ", Éducation comparée. Revue de recherche internationale et comparative en éducation, ${ }^{\circ} 18, \mathrm{pp} .19-38$.

Benoit H. (2014a), « Politiques publiques, professionnalités et langages : Les maillons faibles de la chaîne inclusive », in (Canat S. et Benoit H. (dir.)), dossier «Les dispositifs Itep en devenir », La nouvelle revue de l'adaptation et de la scolarisation, $n^{\circ}$ 67, pp. 181-191.

Benoit H. (2014b), «Les dispositifs inclusifs: freins ou leviers pour l'évolution des pratiques ? », in (Assude T. et Pérez J.-M. (dir.)), La nouvelle revue de l'adaptation et de la scolarisation, $n^{\circ}$ 65, pp. 189-204.

Benoit H. (2014c), «Éducation des jeunes sourds : interférence et hybridation des discours autour de la figure du déni, La nouvelle revue de l'adaptation et de la scolarisation, $n^{\circ} 64$, pp. 117-133.

Benoit H. (2013), «Les impasses de l'inclusion: obstacles théoriques et résistances langagières », in (Assude T. et Pérez J.-M. (dir.)), Pratiques inclusives et savoirs scolaires : paradoxes, contradictions et perspectives, Presses Universitaires de Nancy, pp. 47-59.

Benoit H. (2008), «De la reproduction des pratiques à leur transformation : le défi de la formation des enseignants », in (Gardou C. et Jeanne Y. (dir.)), dossier «Faire culture commune », Reliance, $\mathrm{n}^{\circ}$ 27, pp. 99-104.

Benoit H. (2005), « Inégalités sociales et traitement ségrégatif de la difficulté scolaire : histoire ou actualité ? », La nouvelle revue de l'AIS, n 31, pp. 91-100.

Benoit H. (2003), « Comment en est-on venu à l'UPI ? Forme et signification d'un dispositif », in Magnin de Cagny C. et Ebersold S. (dir.), dossier "Les UPI, les élèves en situation de handicap dans le second degré », La nouvelle revue de l'AIS, $\mathrm{n}^{\circ} 21$, pp. 33-48.

Bourdieu P. (1979), La distinction. Critique sociale du jugement, Les Éditions de Minuit.

D'Alessio S. (2011), Inclusive education in Italy A critical Analysis of the Policy of Integrazione Scolastica, Sense Publishers, 165 . 
D'Alessio S. (20082009), «30 ans d'Integrazione scolastica en Italie. Réflexions critiques et suggestions sur le développement de l'éducation inclusive en Italie », in (Benoit $\mathrm{H}$. et Plaisance E. (dir.)), «L'éducation en France et dans le monde », La nouvelle revue de l'adaptation et de la scolarisation, hors-série $\mathrm{n}^{\circ} 5$, pp. 35-50.

Delsarte J.F. (2012), «Quel partenariat entre l'enseignement spécialisé et l'enseignement ordinaire ? », La nouvelle revue de l'adaptation et de la scolarisation, ${ }^{\circ}$ 57, pp. 35-44.

Dupont H. (2016), Ni fou, ni gogol! Orientation et vie en ITEP, PUG, 240 p.

Durkheim É. (1977), Les Règles de la méthode sociologique, Presses universitaires de France [1894].

Ebersold S. et Dupont H. (2019), «Évaluation des besoins, importunité scolaire et réinvention de l'inéducable », in (Perez J.-M., Benoit H. et Suau G. (dir.)), «Recherche en éducation et pratiques inclusives », La nouvelle revue Éducation et société inclusives, $\mathrm{n}^{\circ}$ 86, pp. 65-78.

Ebersold S. et Detraux J.-J (2013), «Scolarisation et besoins éducatifs particuliers : enjeux conceptuels et méthodologiques d'une approche polycentrée », Alter. Revue européenne de recherche sur le handicap, vol. 7, $\mathrm{n}^{\circ} 2$ (numéro thématique), pp. 102-115.

Emery R. (2017), «La collaboration multiprofessionnelle dans les contextes d'institution spécialisée. Dimensions thématiques et concepts convoqués pour l'étude des pratiques », $L a$ nouvelle revue de l'adaptation et de la scolarisation, $\mathrm{n}^{\circ}$ 78, pp. 193-209.

Foucault M. (1977), «Le jeu de Michel Foucault», Ornicar? Bulletin périodique du champ freudien, $\mathrm{n}^{\circ}$ 10, juillet 1977, repris in (Defert D. et Ewald F. (eds)) (2001), Dits et écrits II, 1976-1988, Quarto Gallimard, texte ${ }^{\circ}$ 206, pp. 298-329.

Foucault M. (1971), L'ordre du discours, Leçon inaugurale au Collège de France prononcée le 2 décembre 1970, Gallimard, $82 \mathrm{p}$.

Foucault M. (1966), Les mots et les choses, Gallimard, coll. Tel, rééd. 2008, 400 p.

Gateaux J. (2005), «Discours pathologique et distanciation sociale », La nouvelle revue de l'adaptation et de la scolarisation, $\mathrm{n}^{\circ} 31$, pp. 37-53.

Gateaux J. (1990), La débilité légère, une construction idéologique, rééd. 2001, CNRS.

Ianes D. et Demo H. (2013), «Que peut-on apprendre de l'expérience italienne ? Quelles démarches pour améliorer l'inclusion?», La nouvelle revue de l'adaptation et de la scolarisation, $\mathrm{n}^{\circ}$ 61, pp. 109-124.

Medeghini R. et D'Alessio S. (2012), «Contribution des systèmes de soutien au développement de l'éducation inclusive », La nouvelle revue de l'adaptation et de la scolarisation, $\mathrm{n}^{\circ} 57$, pp. 13-24. 
MEN - DGESCO B2-2 (2010), « Scolarisation des élèves handicapés. Dispositif collectif au sein d'un établissement du second degré », Circ. $\mathrm{n}^{\circ}$ 2010-088, 18 juin 2010 : Bulletin officiel $\mathrm{n}^{\circ} 28,15$ juill. 2010 .

MEN - DGESCO A1-3 (2015), «Scolarisation des élèves en situation de handicap. Unités localisées pour l'inclusion scolaire (Ulis), dispositifs pour la scolarisation des élèves en situation de handicap dans le premier et le second degré », Circ. $\mathrm{n}^{\circ}$ 2015-129, 21 août 2015, Bulletin officiel $\mathrm{n}^{\circ} 31,27$ août 2015 .

Plaisance E. (2012), «Special educational needs selon Mary Warnock: autocritique ou reniement », La nouvelle revue de l'adaptation et de la scolarisation, $\mathrm{n}^{\circ}$ 57, pp. 211-217.

Rutherford G. (2012), «In, out or somewhere in between ? Disabled students' and teacher aides' experiences of school », International Journal of Inclusive Éducation.

Sensevy G. (2006), « L'action didactique. Éléments de théorisation », Revue suisse des sciences de l'éducation, $\mathrm{n}^{\circ} 2$, pp. 205-226.

Slee R. (2006). «Limits to and possibilities for educational reform », International Journal of Inclusive Éducation, $\mathrm{n}^{\circ} 10$ (2-3), pp. 109-119.

Thomazet S. (2008), "De l'intégration à l'inclusion. Une nouvelle étape dans l'ouverture de l'école aux différences $\gg$, Le Français aujourd'hui, n 152, pp. 1927.

Thomazet S. (2012), Du handicap aux besoins éducatifs particuliers, Le Français aujourd'hui, 2012/2 177, 11-17.

UNESCO (1994), Déclaration de Salamanque, Unesco.

Vadala G., Medeghini R. et D'Alessio S. (2013), « Analyse critique du processus d'intégration scolaire en Italie: vers une prospective inclusive », in (Perez J.-M. et Assude T. (eds)), Pratiques inclusives et savoirs scolaires : paradoxes, contradictions et perspectives, Presses universitaires de Nancy, pp. 29-46.

Vergnaud G. (2004), «Un cadre général en guise d'introduction », La nouvelle revue de l'AIS, $\mathrm{n}^{\circ} 27$, pp. 7-13.

Watkins A. (2007), L'évaluation dans le cadre de l'inclusion: Politique générale et mise en pratique, Agence européenne pour le développement de l'éducation des personnes présentant des besoins particuliers. 
«EN QUOI LES BESOINS EDUCATIFS PARTICULIERS

PEUVENT A LA FOIS ETRE CONSIDERES PAR CERTAINS

COMME L'INSTRUMENT DU PROCESSUS DE

DISCRIMINATION DES ELEVES EN SITUATION DE

HANDICAP, TANDIS QUE D'AUTRES Y VOIENT AU

CONTRAIRE LE MOYEN LE PLUS EFFICACE DE S'Y OPPOSER

ET DE PROMOUVOIR L'ECOLE INCLUSIVE ? »

\section{Hervé Benoit}

